\title{
Recent insights into the role of ChREBP in intestinal fructose absorption and metabolism
}

\author{
Ho-Jae Lee ${ }^{1} \mathcal{E}$ Ji-Young Cha ${ }^{1,2, *}$ \\ ${ }^{1}$ Department of Biochemistry, Lee Gil Ya Cancer and Diabetes Institute, Gachon University College of Medicine, Incheon $21999,{ }^{2}$ Gachon \\ Medical Institute, Gil Medical Center, Incheon 21565, Korea
}

Fructose in the form of sucrose and high fructose corn syrup is absorbed by the intestinal transporter and mainly metabolized in the small intestine. However, excess intake of fructose overwhelms the absorptive capacity of the small intestine, leading to fructose malabsorption. Carbohydrate response element-binding protein (ChREBP) is a basic helix-loop-helix leucine zipper transcription factor that plays a key role in glycolytic and lipogenic gene expression in response to carbohydrate consumption. While ChREBP was initially identified as a glucose-responsive factor in the liver, recent evidence suggests that ChREBP is essential for fructoseinduced lipogenesis and gluconeogenesis in the small intestine as well as in the liver. We recently identified that the loss of ChREBP leads to fructose intolerance via insufficient induction of genes involved in fructose transport and metabolism in the intestine. As fructose consumption is increasing and closely associated with metabolic and gastrointestinal diseases, a comprehensive understanding of cellular fructose sensing and metabolism via ChREBP may uncover new therapeutic opportunities. In this mini review, we briefly summarize recent progress in intestinal fructose metabolism, regulation and function of ChREBP by fructose, and delineate the potential mechanisms by which excessive fructose consumption may lead to irritable bowel syndrome. [BMB Reports 2018; 51(9): 429-436]

\section{INTRODUCTION}

Fructose is a monosaccharide that is derived mostly from fruits and a few vegetables. It is typically consumed as sucrose, a disaccharide composed of 50\% fructose and $50 \%$ glucose, or as a component of high-fructose corn syrup (HFCS, mostly

${ }^{*}$ Corresponding author. Tel: +82-32-899-6070; Fax: +82-32-8996032; E-mail: jycha1014@gachon.ac.kr

https://doi.org/10.5483/BMBRep.2018.51.9.197

Received 27 July 2018

Keywords: ChREBP, Fructose intolerance, Fructose metabolism, Irritable bowel syndrome, Small intestine containing $\sim 42-55 \%$ fructose and the remainder as glucose). HFCS and sucrose are commonly added as sweeteners to many processed foods and carbonated beverages, such as cakes, cookies, cereals, cured meats, flavored yogurts, and soft and fruit drinks. The use of HFCS has increased compared with that of sucrose because it is relatively inexpensive, enhanced flavor, and long shelf-life (1). In the early of 1970s, HFCS intake was $0.4 \%$ of the calories ingested through caloric sweeteners in the United States, reaching a rate of $42 \%$ in the 2000s (2). In recent years, although worldwide intake of added sugars has decreased or stabilized, but represents $\sim 17 \%$ (300 kcal or $75 \mathrm{~g} /$ day) of total energy intake in the United States, still well above the recommended limit of $10 \%$ (3). The mean total consumption of sugar by Koreans from 2008 to 2011 was $61.4 \mathrm{~g} /$ day, corresponding to $12.8 \%$ of total daily energy intake (4). The sugar consumption of adolescents and young adults was substantially higher, and especially beverage-driven sugar accounted for almost $25 \%$ of the total sugar intake.

The increased consumption of sugar and HFCS is associated with obesity, dyslipidemia, insulin resistance, non-alcoholic fatty liver disease, and diabetes (5-7). Moreover, excessive fructose consumption can overwhelm the absorptive capacity of the small intestine, resulting in incomplete absorption, i.e., fructose malabsorption $(8,9)$. Fructose has the same chemical formula as glucose $\left(\mathrm{C}_{6} \mathrm{H}_{12} \mathrm{O}_{6}\right)$. However, its metabolic effects appear to be more harmful than those of glucose, with excessive fructose intake increasing de novo lipogenesis and serum triglyceride levels (10).

Recently, we reported that glucose sensing transcription factor ChREBP (carbohydrate response element-binding protein) plays a pivotal role in the expression of fructose-induced transporter and the insufficient induction of glucose transporter 5 (GLUT5) in small intestine leads to fructose malabsorption. In this review, we principally focus on fructose absorption and metabolism in the small intestine, the regulation of ChREBP by fructose, and extend the potential mechanisms by which excessive fructose consumption may contribute to irritable bowel syndrome (IBS). 


\section{FRUCTOSE ABSORPTION AND CONCENTRATIONS IN THE BLOOD}

Fructose is primarily absorbed in the small intestine via GLUT5 (encoded by Slc2A5: $K_{m}, 6-14 \mathrm{mM}$ ), the main fructose transporter in mammals $(11,12)$ in an energy-independent process, with carrier-limited absorptive capacity (13). GLUT5 is expressed in the apical lumen facing the border of intestinal mucosa and the basolateral enterocyte membranes $(14,15)$ (Fig. 1). The liver is assumed as a primary site for fructose metabolism, but most ingested fructose is initially metabolized by the small intestine. More than $90 \%$ of fructose is actively metabolized into glucose and lactate in the small intestine and exported via portal blood (16). From the portal plasma, fructose is then efficiently taken up by the liver with limited amounts escaping the hepatic metabolism and entering the systemic circulation. The changes in fructose concentrations in the luminal, portal and peripheral blood reflect metabolic flux. Consumption of $65 \%$ fructose diets by young rats results in 26 $\mathrm{mM}$ fructose concentrations in the intestinal lumen (17). Portal and systemic fructose concentrations in rodents and other mammals are typically lower than $0.1 \mathrm{mM}$ when fasting or consuming fructose-free, low-sucrose diets, and increase to $\sim 1 \mathrm{mM}$ following consumption of diets containing $40 \%$ to $60 \%$ fructose $(18,19)$. Meanwhile, glucose is transported by sodium-glucose cotransporter 1 (SGLT1) on the apical membrane from the intestinal lumen into the enterocytes, and further into the portal blood by GLUT2 on the basolateral membrane (20) (Fig. 1). The glucose diffuses from the small intestine into the body mostly passively.

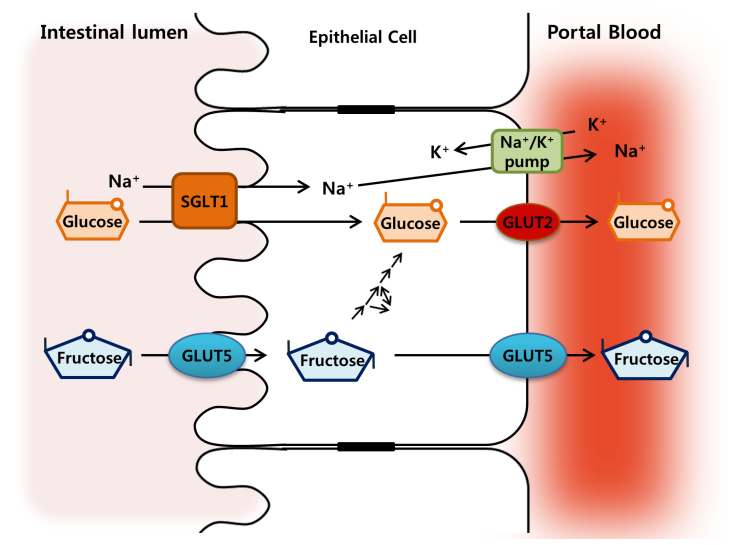

Fig. 1. Models for glucose and fructose transport across the intestinal epithelium. Glucose is transported into the enterocyte across the brush border membrane by the sodium glucose cotransporter 1 (SGLT1) and released across the basolateral membrane down the concentration gradients by glucose transporter 2 (GLUT2). The $\mathrm{Na}^{+} / \mathrm{K}^{+}$pump in the basolateral membrane maintains the functional gradient of SGLT1. Fructose is transported through the brush border membrane and extruded basolaterally by facilitated diffusion via GLUT5.

\section{SMALL INTESTINE AS THE PRIMARY ORGAN FOR FRUCTOSE METABOLISM}

Most previous studies have shown that the ingested fructose is mainly metabolized in the liver by fructokinase and aldolase B. However, recent evidence shows that the small intestine also expresses fructolytic and gluconeogenic enzymes, similar to liver, and thus plays an important role in fructose metabolism (Fig. 2). Upon entering the intestinal epithelial cells via GLUT5, fructose is rapidly phosphorylated to fructose-1-phosphate (F-1-P) by fructokinase (KHK, ketohexokinase). KHK is a unique hexokinase characterized by the absence of feedback inhibition resulting in intracellular phosphate depletion and rapid generation of uric acid due to activation of AMP deaminase $(21,22)$. Although direct evidence suggesting uric acid synthesis upon fructose feeding in the intestine is unavailable, the small intestine is an important organ for uric acid production (23). KHK exists in two splicing isoforms, KHK-A and KHK-C, with KHK-C representing the major phosphorylating enzyme due to its lower $K_{m}(24)$. KHK-C is primarily expressed in intestine, liver, kidney, and pancreas, whereas a splicing variant KHK-A is more ubiquitous (25). Fructose metabolism bypasses significant regulatory steps in the glucose metabolic pathway: glucose-6-phosphate (G-6-P) generation by hexokinase and fructose-1,6-bisphosphate generation by phosphofructokinase (26). F-1-P is then directly cleaved into three-carbon units, dihydroxyacetone phosphate (DHAP) and glyceraldehyde by aldolase B (27). Glyceraldehyde, unlike the products of aldolase in glycolysis, must be phosphorylated into

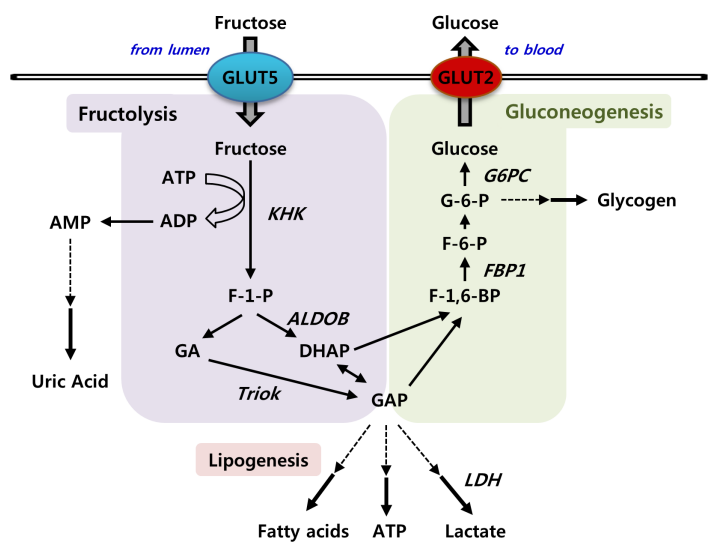

Fig. 2. Fructose metabolism in the small intestine. Fructose is efficiently metabolized by ketohexokinase (KHK) into fructose-1phosphate $(\mathrm{F}-1-\mathrm{P})$. F-1-P is then cleaved by aldolase B (AldoB) into dihydroxyacetone phosphate (DHAP) and glyceraldehyde. Glyceraldehyde is phosphorylated by triokinase (TrioK) to provide glyceraldehyde 3-phosphate (GAP). The triose phosphates derived from fructolysis are resynthesized into glucose via gluconeogenesis or further metabolized into lactate or acetyl-CoA, which are oxidized or used for lipogenesis. 
glyceraldehyde-3-phosphate by triokinase (TrioK). DHAP and glyceraldehyde-3-phosphate are identical to glycolytic intermediates and enter the gluconeogenic pathway for glucose or further catabolized via the lower glycolytic pathway to lactate $(28,29)$.

The disposition of fructose-derived carbon among the major metabolic pathways depends on the overall nutritional and endocrine status of the animal as well as the status of key regulatory checkpoints in intermediary metabolism. Fructose is absorbed from the small intestine, where it is phosphorylated and cleaved into three-carbon units, and converted into glucose and organic acids. A recent study using isotope tracing and mass spectrometry has shown that in mice, the small intestine converts $\sim 42 \%, \sim 20 \%$ and $\sim 10 \%$ of the fructose to glucose, lactate, and alanine, respectively (16). Only $\sim 14 \%$ of fructose is released into the portal blood by the small intestine. Additionally, $\sim 3 \%$ of fructose is converted into other organic acids such as glycerate, TCA intermediates, and amino acids. The passage of unmetabolized fructose through the small intestine to the liver depends on dose rate. Low doses of fructose are nearly entirely cleared by the small intestine, but high doses of fructose overwhelm intestinal fructose absorption and clearance capacity. The extra fructose is digested by the liver and colonic microbiota. Notably, intestinal fructose absorption and metabolism are augmented both by prior exposure to fructose and by feeding (16).

\section{REGULATION OF ChREBP ACTIVITY BY GLUCOSE AND FRUCTOSE}

ChREBP is a basic helix-loop-helix leucine zipper (bHLH-ZIP) transcription factor initially discovered as a deleted gene product in Williams-Beuren syndrome, WBSCR14 and later found to recognize the carbohydrate response element (ChoRE) within the promoter of the liver-type pyruvate kinase (LPK) $(30,31)$. ChREBP forms a heterodimeric complex with Max-like protein $X(\mathrm{MlX})$ and activates the transcription of ChoRE-containing target genes in response to glucose and fructose (32-34).

\section{ChREBP regulation by glucose}

The transcriptional activity of ChREBP is regulated by its phosphorylation status, subcellular localization, glucose metabolites, post-translational modification, and protein stability. ChREBP contains two major cAMP-activated protein kinase (PKA) phosphorylation sites, $\operatorname{Ser}^{196}$ (P1) and $\mathrm{Thr}^{666}$ (P3), which are regulated by CAMP and glucose. When glucose levels are low, the phosphorylation of ChREBP by PKA inactivates DNA-binding activity, whereas protein phosphatase 2A (PP2A) dephosphorylates P1 site of ChREBP, which results in stimulation of ChREBP import into the nucleus. Following nuclear translocation of ChREBP, glucose signaling activates the transcriptional activity of ChREBP by dephosphorylation of the P3 site by PP2A $(31,35)$. Under conditions of low energy,
AMP-activated protein kinase (AMPK) phosphorylates P1 sites of ChREBP, resulting in its transcriptional inactivation by reducing DNA binding $(36,37)$.

ChREBP is activated by glucose metabolites, such as G-6-P, xylulose-5-phosphate (Xu-5-P), and fructose-2,6-bisphosphate (F-2,6-BP). The phosphorylation of glucose to G-6-P catalyzed by glucokinase is the first step of glycolysis and leads to the production of essential metabolites for ChREBP activation. Dentin $\mathrm{R}$ et al. showed that G-6-P synthesis via over-expression of glucose-6-phosphate dehydrogenase is required for glucose-induced activation of ChREBP (38). Xu-5-P, a metabolite of the pentose phosphate pathway, activates PP2A, which in turn dephosphorylates P1 and P3 sites of ChREBP and hence, facilitates ChREBP nuclear translocation and activation $(39,40)$. F-2,6-BP, a key regulator of glycolysis and gluconeogenesis, has been implicated in ChREBP activation. Arden $\mathrm{C}$ et al. demonstrated that the enzymatic depletion of F-2,6-BP inhibits glucose-induced recruitment of ChREBP to the promoter of target genes (41).

ChREBP has two isoforms, ChREBP- $\alpha$ and ChREBP- $\beta$, and each ChREBP isoform complexed with Mlx regulates ChREBP target gene expression (42). ChREBP- $\alpha$ contains a low-glucose inhibitory domain (LID) and a glucose-response activation conserved element (GRACE) $(42,43)$. The glucose responsiveness of ChREBP is associated with the intramolecular inhibition between LID and GRACE. Under low glucose conditions, the transcriptional activity of GRACE is inhibited by the LID, whereas high glucose conditions release this inhibition (43). In addition, upon glucose stimulation, ChREBP- $\alpha$ is translocated from the cytosol to the nucleus, whereas ChREBP- $\beta$, which lacks LID and is constitutively active, is mainly localized in the nucleus. Glucose-activated ChREBP- $\alpha$ also induces the transcription of ChREBP- $\beta$ suggesting a feed-forward mechanism in which ChREBP- $\alpha$ is first activated by glucose metabolism and, in turn, stimulates the expression of ChREBP- $\beta$, a more potent activator of ChREBP transcriptional targets (42). In addition, ChREBPinteracting proteins, such as 14-3-3 proteins, CRM1, and importins play an essential role in subcellular trafficking of ChREBP in response to variations in nutritional status (44-46).

The transcriptional activity of ChREBP is also modulated via post-translational modification. Bricambert J et al. showed that glucose-activated histone acetyltransferase (HAT) coactivator p300 acetylates ChREBP and increases its transcriptional activity by enhancing its binding to target gene promoters (47). In addition, high glucose concentrations activate O-linked $\mathrm{N}$-acetylglucosamine transferase (OGT)-induced O-glycosylation of ChREBP and increase DNA binding of ChREBP. The O-glycation of ChREBP is suggested to decrease the ubiquitinmediated degradation of $\operatorname{ChREBP}(48,49)$.

\section{ChREBP regulation by fructose}

Fructose also activates ChREBP transcriptional activity by increased DNA binding, phosphorylation via Xu-5-P, 
O-glycosylation and acetylation (41, 50, 51). Recently, Kim M et al. showed that high fructose diet (HFrD) markedly induces hepatic and intestinal ChREBP- $\beta$ expression, followed by increased glycolytic, fructolytic, and lipogenic gene expression $(52,53)$. We also showed that the intestinal protein but not mRNA levels of ChREBP were increased by HFrD, accompanied by increased fructolytic and gluconeogenic gene expression (54).

\section{ChREBP TARGET GENES}

ChREBP is highly expressed in key metabolic tissues, including liver, adipose tissue, small intestine, kidney, and pancreatic $\beta$-cells, where it regulates carbohydrate metabolism in an insulin-independent manner $(55,56)$. Most of ChREBP targets have been identified in hepatocytes, adipocytes, and pancreatic $\beta$ cells, where it contributes to the regulation of glucose sensing and/or de novo fatty acid synthesis. Since its discovery, ChREBP has proven to be a key regulator of genes involved in glycolysis and pentose phosphate pathway (Glut2, Pklr, G6pdh), lipogenesis (Acaca, Fasn, Scd1, Elovl6), and triglyceride formation (Gpdh, Dgat2). ChREBP also regulates some genes involved in fructolysis (Khk, Aldolase $B$, Triokinase), gluconeogenesis (G6pase, Fbp1), and hormone and hormone receptors (fibroblast growth factor 21 , glucagon receptor, and adiponectin receptor 2) (34, 55, 57-63). ChREBP was first identified as a transcriptional activator of its target genes, but it also acts as a transcriptional repressor $(55,57,64,65)$.

Although ChREBP is primarily a glucose-responsive factor, recent observations in different animal models suggest a crucial role in fructose metabolism and metabolic disease. In rats, high-fructose feeding, when compared with glucose, was associated with increased ChREBP activity and the expression of its target genes (50). Accordingly, mice fed with HFrD showed an increase in hepatocellular carbohydrate metabolites, expression of ChREBP target genes, and hepatic steatosis, due to hepatic ChREBP activation (52). Moreover, ChREBP is essential for fructose-induced increases in circulating FGF21, which is required for a normal hepatic metabolic response to fructose consumption, and the absence of FGF21 leads to liver disease in mice exposed to HFrD (66).

\section{INTESTINAL ChREBP AS AN ESSENTIAL MEDIATOR FOR FRUCTOSE TOLERANCE}

In the intestine, fructose rapidly induces genes involved in its uptake and catabolism. GLUT5 plays a major role in regulating the fructose entry in our body. Deletion of GLUT5 reduces intestinal fructose absorption as well as serum fructose concentration by $>80 \%$ (67). GLUT5 mRNA and protein abundance are markedly increased within a few hours of fructose consumption, resulting in enhanced fructose absorption $(14,67)$. Patel $C$ et al. showed that fructose absorption via GLUT5 and KHK-mediated fructose metabolism are required for the regulation of intestinal fructolytic and gluconeogenic enzymes (68). The feedforward regulation of fructolytic and gluconeogenic enzymes may proactively enhance the intestinal ability to process the anticipated increases in dietary fructose concentrations. It has been reported that ChREBP directly upregulates hepatic Khk following high fructose intake (51). Recently, we have reported that ChREBP is a key transcription factor in fructose-mediated upregulation of fructose transporter (Glut5), fructolytic enzymes (Khk, AldoB, TrioK, Ldh), and gluconeogenic enzymes (G6pc and Fbp1) in the intestine (Fig. 3) (54). We demonstrated that ChREBP directly regulates Glut5 gene expression through functional ChREBP-ChoRE (-2165 - 2149 bp) interaction (54).

The significant role of ChREBP in fructose metabolism is supported by the observations of rapid weight loss, hypothermia, and moribund state observed in global ChREBP knockout mice exposed to diets containing sucrose or fructose $(54,55)$. The diminished expression of enzymes required for fructose entry into glycolysis, such as aldolase B and KHK in the liver of ChREBP KO mouse was proposed as the reason for the fructose intolerance $(52,56)$. However, recent reports suggest

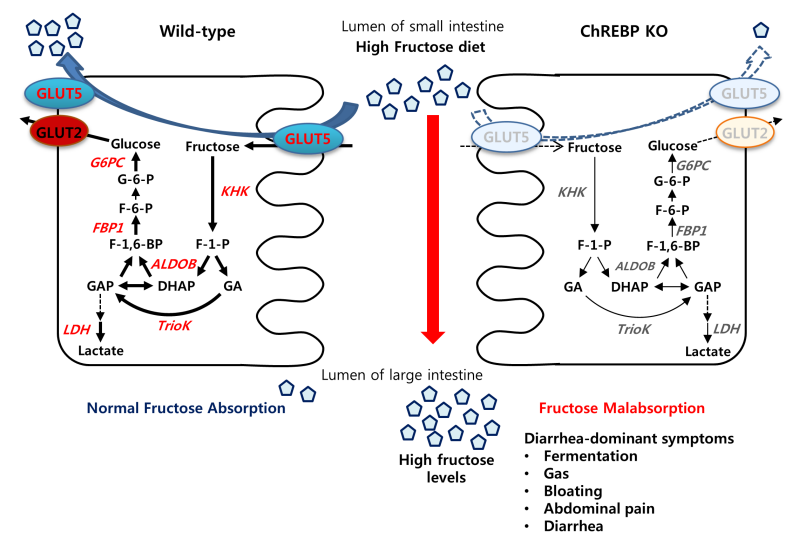

Fig. 3. Model showing the effects of ChREBP deletion on fructose transport and metabolism upon high-fructose diet consumption. Upon high fructose ingestion, genes involved in fructose transport (Glut5), fructolysis (Khk, $A / d o B, T r i o k$, and $L d h)$, and gluconeogenesis (G6pc and Fbp1) are significantly increased in the intestinal cells. However, ChREBP knockout mice show impaired induction of GLUT5 as well as fructolytic and glucogenic enzymes in the intestine. As a result, HFrD-fed ChREBP knockout mice develop diarrhea-dominant IBS symptoms, such as gas, bloating, abdominal pain, or diarrhea. Genes significantly increased by high fructose in WT mice but not in ChREBP knockout mice are indicated in red color. ALDOB, aldolase B; ChREBP, carbohydrate response element-binding protein; DHAP, dihydroxyacetone phosphate; F-1-P, fructose-1-phosphate; F-1,6-BP, fructose-1,6-bisphosphate; FBP1, fructose-1,6-bisphosphatase; GA, glyceraldehyde; GAP, glyceraldehyde-3-phosphate; GLUT2, glucose transporter 2; GLUT5, glucose transporter 5; G-6-P, glucose-6phosphate; G6PC, glucose-6-phosphatase; KHK, fructokinase; LDH, lactate dehydrogenase; TrioK, triokinase. 
that intestinal, but not hepatic ChREBP is essential for fructose tolerance $(53,54,69)$. ChREBP knockout mice are fructose intolerant mainly due to a decreased capacity for fructose absorption following insufficient induction of GLUT5 upon high fructose ingestion (Fig. 3). As a result, a high concentration of fructose in the gut leads to water influx into the lumen due to osmotic pressure, and rapid propulsion of bowel contents into the colon. Colonic bacterial fermentation of unabsorbed fructose may generate gas, bloating, or diarrhea and results in decreased food intake.

\section{ChREBP DEFICIENCY LEADS TO FRUCTOSE MALABSORPTION AND IRRITABLE BOWEL DISEASE}

IBS, one of the most frequent functional gastrointestinal disorders (FGIDs), is characterized by symptoms of abdominal pain, bloating, and altered bowel habits, which include diarrhea, constipation, or both. The pathophysiology of IBS remains incompletely understood, but factors such as altered gastrointestinal (Gl) motility, abnormal sensory function of the intestine, malabsorption of food products, bacterial overgrowth in the small intestine and psychosocial factors may play a role (70). In normal physiology, fructose is absorbed across the intestinal mucosa by facilitated diffusion via GLUT5, and its absorption capacity is limited (71). Therefore excess dietary intake of fructose can quickly overwhelm the absorptive capacity of the small intestine, leading to incomplete absorption and resulting in fructose malabsorption (72). Fructose malabsorption, previously described as dietary fructose intolerance, was first described in 1978 based on a breath test report of fructose malabsorption (73) and later described in IBS patients $(74,75)$. The unabsorbed fructose represents an osmotic load propelling the sugar into the colon, where contact with anaerobic microbiota causes fermentation and production of gas, abdominal bloating, and diarrhea (76). However, the precise pathophysiology leading to fructose malabsorption is not clear. Barone $\mathrm{S}$ et al. have previously shown that HFrD-fed GLUT5 knockout mouse model exhibits a decreased absorption of dietary fructose, with consequent distension of the large intestine with fluid and gas. The model presented the hallmarks of fructose malabsorption (67). Recently, we and others demonstrated that HFrD-fed ChREBP knockout mice exhibit a severely distended cecum, with proximal colon containing both gas and fluid content, and severe diarrhea, suggesting incomplete fructose absorption $(53,54,69)$.

\section{CONCLUSIONS AND PERSPECTIVES}

In this review, we summarized the role of ChREBP in fructose absorption and metabolism, particularly in the small intestine, discussed recent findings involving ChREBP knockout mice, manifesting diarrhea-predominant IBS symptoms following exposure to HFrD. The underlying pathophysiology of IBS is complex and has yet to be fully understood. The animal model facilitates the investigation of the pathogenesis of IBS without the risks associated with a human study. Until now, the exact mechanism responsible for IBS was limited by the lack of animal models with high face validity. The generation of IBS animal model is mostly based on induction of psychological stress. Thus, our recent study of ChREBP knockout mice fed with $\mathrm{HFrD}$ may represent an appropriate model for the understanding of the mechanisms of IBS, particularly diarrhea-predominant IBS caused by fructose malabsorption. Furthermore, investigations into the changes associated with the intestinal microbiota may elucidate the pathophysiology of IBS and provide a new therapeutic strategy.

\section{ACKNOWLEDGEMENTS}

This work was supported by grants awarded to J-Y Cha (NRF-2016R1A2B4015866) and H-J Lee (NRF-2016R1D1A1B 03935001) by the Basic Science Research Program through the National Research Foundation of Korea (NRF) funded by the Ministry of Education, Science and Technology. We apologize to colleagues whose work could not be cited due to space limitations.

\section{CONFLICTS OF INTEREST}

The authors have no conflicting interests.

\section{REFERENCES}

1. Hanover LM and White JS (1993) Manufacturing, composition, and applications of fructose. Am J Clin Nutr 58, 724S-732S

2. Bray GA, Nielsen SJ and Popkin BM (2004) Consumption of high-fructose corn syrup in beverages may play a role in the epidemic of obesity. Am J Clin Nutr 79, 537-543

3. Powell ES, Smith-Taillie LP and Popkin BM (2016) Added Sugars Intake Across the Distribution of US Children and Adult Consumers: 1977-2012. J Acad Nutr Diet 116, 1543-1550 e1541

4. Lee H-S, Kwon S-o, Yon M et al (2014) Dietary total sugar intake of Koreans: Based on the Korea National Health and Nutrition Examination Survey (KNHANES), 2008-2011. J Nutr Health 47, 268-276

5. Elliott SS, Keim NL, Stern JS, Teff K and Havel PJ (2002) Fructose, weight gain, and the insulin resistance syndrome. Am J Clin Nutr 76, 911-922

6. Haidari M, Leung N, Mahbub F et al (2002) Fasting and postprandial overproduction of intestinally derived lipoproteins in an animal model of insulin resistance. Evidence that chronic fructose feeding in the hamster is accompanied by enhanced intestinal de novo lipogenesis and ApoB48-containing lipoprotein overproduction. J Biol Chem 277, 31646-31655

7. Stanhope KL (2012) Role of fructose-containing sugars in the epidemics of obesity and metabolic syndrome. Annu 
Rev Med 63, 329-343

8. Jones HF, Butler RN and Brooks DA (2011) Intestinal fructose transport and malabsorption in humans. Am J Physiol Gastrointest Liver Physiol 300, G202-206

9. Heizer WD, Southern S and McGovern S (2009) The role of diet in symptoms of irritable bowel syndrome in adults: a narrative review. J Am Diet Assoc 109, 1204-1214

10. Le KA, Ith M, Kreis R et al (2009) Fructose overconsumption causes dyslipidemia and ectopic lipid deposition in healthy subjects with and without a family history of type 2 diabetes. Am J Clin Nutr 89, 1760-1765

11. Thorens B and Mueckler M (2010) Glucose transporters in the 21st Century. Am J Physiol Endocrinol Metab 298, E141-145

12. Burant CF, Takeda J, Brot-Laroche E, Bell Gl and Davidson NO (1992) Fructose transporter in human spermatozoa and small intestine is GLUT5. J Biol Chem 267, 14523-14526

13. Corpe CP, Burant CF and Hoekstra JH (1999) Intestinal fructose absorption: clinical and molecular aspects. J Pediatr Gastroenterol Nutr 28, 364-374

14. Castello A, Guma A, Sevilla L et al (1995) Regulation of GLUT5 gene expression in rat intestinal mucosa: regional distribution, circadian rhythm, perinatal development and effect of diabetes. Biochem J 309, 271-277

15. Blakemore SJ, Aledo JC, James J, Campbell FC, Lucocq JM and Hundal HS (1995) The GLUT5 hexose transporter is also localized to the basolateral membrane of the human jejunum. Biochem J 309, 7-12

16. Jang C, Hui S, Lu W et al (2018) The Small Intestine Converts Dietary Fructose into Glucose and Organic Acids. Cell Metab 27, 351-361 e353

17. Ferraris RP, Yasharpour S, Lloyd KC, Mirzayan R and Diamond JM (1990) Luminal glucose concentrations in the gut under normal conditions. Am J Physiol 259, G822-837

18. Patel C, Sugimoto K, Douard V et al (2015) Effect of dietary fructose on portal and systemic serum fructose levels in rats and in KHK $-/-$ and GLUT5 $-/-$ mice. Am J Physiol Gastrointest Liver Physiol 309, G779-790

19. Douard V and Ferraris RP (2008) Regulation of the fructose transporter GLUT5 in health and disease. Am J Physiol Endocrinol Metab 295, E227-237

20. Gorboulev V, Schurmann A, Vallon V et al (2012) $\mathrm{Na}(+)$-D-glucose cotransporter SGLT1 is pivotal for intestinal glucose absorption and glucose-dependent incretin secretion. Diabetes 61, 187-196

21. Woods HF, Eggleston LV and Krebs HA (1970) The cause of hepatic accumulation of fructose 1-phosphate on fructose loading. Biochem J 119, 501-510

22. van den Berghe $G$, Bronfman $M$, Vanneste $R$ and Hers HG (1977) The mechanism of adenosine triphosphate depletion in the liver after a load of fructose. A kinetic study of liver adenylate deaminase. Biochem J 162, 601-609

23. Yun $Y$, Yin H, Gao Z et al (2017) Intestinal tract is an important organ for lowering serum uric acid in rats. PLoS One 12, e0190194

24. Diggle CP, Shires M, McRae C et al (2010) Both isoforms of ketohexokinase are dispensable for normal growth and development. Physiol Genomics 42A, 235-243

25. Ishimoto T, Lanaspa MA, Le MT et al (2012) Opposing effects of fructokinase $\mathrm{C}$ and $\mathrm{A}$ isoforms on fructoseinduced metabolic syndrome in mice. Proc Natl Acad Sci U S A 109, 4320-4325

26. Mayes PA (1993) Intermediary metabolism of fructose. Am J Clin Nutr 58, 754S-765S

27. Heinz F, Lamprecht W and Kirsch J (1968) Enzymes of fructose metabolism in human liver. J Clin Invest 47, 1826-1832

28. Sun SZ and Empie MW (2012) Fructose metabolism in humans - what isotopic tracer studies tell us. Nutr Metab (Lond) 9, 89

29. Douard V and Ferraris RP (2013) The role of fructose transporters in diseases linked to excessive fructose intake. J Physiol 591, 401-414

30. de Luis O, Valero MC and Jurado LA (2000) WBSCR14, a putative transcription factor gene deleted in WilliamsBeuren syndrome: complete characterisation of the human gene and the mouse ortholog. Eur J Hum Genet 8 , 215-222

31. Yamashita H, Takenoshita M, Sakurai M et al (2001) A glucose-responsive transcription factor that regulates carbohydrate metabolism in the liver. Proc Natl Acad Sci U S A 98, 9116-9121

32. Shih HM, Liu Z and Towle HC (1995) Two CACGTC motifs with proper spacing dictate the carbohydrate regulation of hepatic gene transcription. J Biol Chem 270, 21991-21997

33. Stoeckman AK, Ma L and Towle HC (2004) Mlx is the functional heteromeric partner of the carbohydrate response element-binding protein in glucose regulation of lipogenic enzyme genes. J Biol Chem 279, 15662-15669

34. Ma L, Robinson LN and Towle HC (2006) ChREBP*Mlx is the principal mediator of glucose-induced gene expression in the liver. J Biol Chem 281, 28721-28730

35. Kawaguchi T, Takenoshita M, Kabashima T and Uyeda $K$ (2001) Glucose and cAMP regulate the L-type pyruvate kinase gene by phosphorylation/dephosphorylation of the carbohydrate response element binding protein. Proc Natl Acad Sci U S A 98, 13710-13715

36. Kawaguchi T, Osatomi K, Yamashita H, Kabashima T and Uyeda K (2002) Mechanism for fatty acid "sparing" effect on glucose-induced transcription: regulation of carbohydrate-responsive element-binding protein by AMPactivated protein kinase. J Biol Chem 277, 3829-3835

37. Sato $S$, Jung $H$, Nakagawa $T$ et al (2016) Metabolite Regulation of Nuclear Localization of Carbohydrateresponse Element-binding Protein (ChREBP): ROLE OF AMP AS AN ALLOSTERIC INHIBITOR. J Biol Chem 291, 10515-10527

38. Dentin R, Tomas-Cobos L, Foufelle F et al (2012) Glucose 6-phosphate, rather than xylulose 5-phosphate, is required for the activation of ChREBP in response to glucose in the liver. J Hepatol 56, 199-209

39. Kabashima T, Kawaguchi T, Wadzinski BE and Uyeda K (2003) Xylulose 5-phosphate mediates glucose-induced lipogenesis by xylulose 5-phosphate-activated protein phosphatase in rat liver. Proc Natl Acad Sci U S A 100, 5107-5112 
40. lizuka K, Wu W, Horikawa Y and Takeda J (2013) Role of glucose-6-phosphate and xylulose-5-phosphate in the regulation of glucose-stimulated gene expression in the pancreatic beta cell line, INS-1E. Endocr J 60, 473-482

41. Arden C, Tudhope SJ, Petrie JL et al (2012) Fructose 2,6-bisphosphate is essential for glucose-regulated gene transcription of glucose-6-phosphatase and other ChREBP target genes in hepatocytes. Biochem J 443, 111-123

42. Herman MA, Peroni OD, Villoria J et al (2012) A novel ChREBP isoform in adipose tissue regulates systemic glucose metabolism. Nature 484, 333-338

43. Li MV, Chang B, Imamura M, Poungvarin N and Chan L (2006) Glucose-dependent transcriptional regulation by an evolutionarily conserved glucose-sensing module. Diabetes 55, 1179-1189

44. Sakiyama H, Wynn RM, Lee WR et al (2008) Regulation of nuclear import/export of carbohydrate response element-binding protein (ChREBP): interaction of an alpha-helix of ChREBP with the 14-3-3 proteins and regulation by phosphorylation. J Biol Chem 283, 24899-24908

45. Fukasawa M, Ge Q, Wynn RM, Ishii S and Uyeda K (2010) Coordinate regulation/localization of the carbohydrate responsive binding protein (ChREBP) by two nuclear export signal sites: discovery of a new leucine-rich nuclear export signal site. Biochem Biophys Res Commun 391, 1166-1169

46. Ge Q, Nakagawa T, Wynn RM, Chook YM, Miller BC and Uyeda K (2011) Importin-alpha protein binding to a nuclear localization signal of carbohydrate response element-binding protein (ChREBP). J Biol Chem 286, 28119-28127

47. Bricambert J, Miranda J, Benhamed F, Girard J, Postic C and Dentin R (2010) Salt-inducible kinase 2 links transcriptional coactivator p300 phosphorylation to the prevention of ChREBP-dependent hepatic steatosis in mice. J Clin Invest 120, 4316-4331

48. Guinez C, Filhoulaud G, Rayah-Benhamed F et al (2011) O-GlcNAcylation increases ChREBP protein content and transcriptional activity in the liver. Diabetes 60, 1399-1413

49. Ido-Kitamura Y, Sasaki T, Kobayashi M et al (2012) Hepatic FoxO1 integrates glucose utilization and lipid synthesis through regulation of Chrebp O-glycosylation. PLoS One 7, e47231

50. Koo HY, Miyashita M, Cho BH and Nakamura MT (2009) Replacing dietary glucose with fructose increases ChREBP activity and SREBP-1 protein in rat liver nucleus. Biochem Biophys Res Commun 390, 285-289

51. Lanaspa MA, Sanchez-Lozada LG, Cicerchi C et al (2012) Uric acid stimulates fructokinase and accelerates fructose metabolism in the development of fatty liver. PLoS One 7, e47948

52. Kim MS, Krawczyk SA, Doridot L et al (2016) ChREBP regulates fructose-induced glucose production independently of insulin signaling. J Clin Invest 126, 4372-4386

53. Kim M, Astapova II, Flier SN et al (2017) Intestinal, but not hepatic, ChREBP is required for fructose tolerance. $\mathrm{JCl}$ Insight 2, e96703

54. Oh AR, Sohn S, Lee J et al (2018) ChREBP deficiency leads to diarrhea-predominant irritable bowel syndrome.
Metabolism 85, 286-297

55. lizuka K, Bruick RK, Liang G, Horton ID and Uyeda K (2004) Deficiency of carbohydrate response elementbinding protein (ChREBP) reduces lipogenesis as well as glycolysis. Proc Natl Acad Sci U S A 101, 7281-7286

56. Uyeda K and Repa JJ (2006) Carbohydrate response element binding protein, ChREBP, a transcription factor coupling hepatic glucose utilization and lipid synthesis. Cell Metab 4, 107-110

57. Jeong YS, Kim D, Lee YS et al (2011) Integrated expression profiling and genome-wide analysis of ChREBP targets reveals the dual role for ChREBP in glucoseregulated gene expression. PLoS One 6, e22544

58. Poungvarin N, Chang B, Imamura M et al (2015) Genome-Wide Analysis of ChREBP Binding Sites on Male Mouse Liver and White Adipose Chromatin. Endocrinology 156, 1982-1994

59. Bae JS, Oh AR, Lee HJ, Ahn YH and Cha JY (2016) Hepatic Elovl6 gene expression is regulated by the synergistic action of ChREBP and SREBP-1c. Biochem Biophys Res Commun 478, 1060-1066

60. lizuka K, Takeda J and Horikawa Y (2009) Glucose induces FGF21 mRNA expression through ChREBP activation in rat hepatocytes. FEBS Lett 583, 2882-2886

61. Pedersen KB, Zhang P, Doumen $C$ et al (2007) The promoter for the gene encoding the catalytic subunit of rat glucose-6-phosphatase contains two distinct glucoseresponsive regions. Am J Physiol Endocrinol Metab 292, E788-801

62. Shin E, Bae JS, Han JY et al (2016) Hepatic DGAT2 gene expression is regulated by the synergistic action of ChREBP and SP1 in HepG2 cells. Anim Cells Syst 20, 7-14

63. lizuka K, Tomita R, Takeda J and Horikawa Y (2012) Rat glucagon receptor mRNA is directly regulated by glucose through transactivation of the carbohydrate response element binding protein. Biochem Biophys Res Commun $417,1107-1112$

64. Noordeen NA, Khera TK, Sun G et al (2010) Carbohydrateresponsive element-binding protein (ChREBP) is a negative regulator of ARNT/HIF-1beta gene expression in pancreatic islet beta-cells. Diabetes 59, 153-160

65. Boergesen M, Poulsen L, Schmidt SF, Frigerio F, Maechler $P$ and Mandrup S (2011) ChREBP mediates glucose repression of peroxisome proliferator-activated receptor alpha expression in pancreatic beta-cells. J Biol Chem 286, 13214-13225

66. Fisher FM, Kim M, Doridot $L$ et al (2017) A critical role for ChREBP-mediated FGF21 secretion in hepatic fructose metabolism. Mol Metab 6, 14-21

67. Barone S, Fussell SL, Singh AK et al (2009) Slc2a5 (Glut5) is essential for the absorption of fructose in the intestine and generation of fructose-induced hypertension. J Biol Chem 284, 5056-5066

68. Patel C, Douard V, Yu S, Tharabenjasin P, Gao N and Ferraris RP (2015) Fructose-induced increases in expression of intestinal fructolytic and gluconeogenic genes are regulated by GLUT5 and KHK. Am J Physiol Regul Integr Comp Physiol 309, R499-509

69. Kato T, lizuka K, Takao K, Horikawa Y, Kitamura T and 
Takeda J (2018) ChREBP-Knockout Mice Show Sucrose Intolerance and Fructose Malabsorption. Nutrients 10, 340

70. Camilleri M (2001) Management of the irritable bowel syndrome. Gastroenterology 120, 652-668

71. Riby JE, Fujisawa $T$ and Kretchmer N (1993) Fructose absorption. Am J Clin Nutr 58, 748S-753S

72. Gibson PR, Newnham E, Barrett JS, Shepherd SJ and Muir JG (2007) Review article: fructose malabsorption and the bigger picture. Aliment Pharmacol Ther 25, 349-363

73. Andersson DE and Nygren A (1978) Four cases of long-standing diarrhoea and colic pains cured by fructose- free diet-a pathogenetic discussion. Acta Med Scand 203, 87-92

74. Ravich WJ, Bayless TM and Thomas M (1983) Fructose: incomplete intestinal absorption in humans. Gastroenterology 84, 26-29

75. Rumessen JJ and Gudmand-Hoyer E (1988) Functional bowel disease: malabsorption and abdominal distress after ingestion of fructose, sorbitol, and fructose-sorbitol mixtures. Gastroenterology 95, 694-700

76. Choi YK, Johlin FC Jr, Summers RW, Jackson $M$ and Rao SS (2003) Fructose intolerance: an under-recognized problem. Am J Gastroenterol 98, 1348-1353 